\title{
Existence and Uniqueness of Solution for Perturbed Nonautonomous Systems with Nonuniform Exponential Dichotomy
}

\author{
Yong-Hui Xia, ${ }^{1}$ Xiaoqing Yuan, ${ }^{1}$ Kit Ian Kou, ${ }^{2}$ and Patricia J. Y. Wong ${ }^{3}$ \\ ${ }^{1}$ Department of Mathematics, Zhejiang Normal University, Jinhua 321004, China \\ ${ }^{2}$ Department of Mathematics, Faculty of Science and Technology, University of Macau, Avenue Padre Tomás Pereira Taipa, Macau, \\ China \\ ${ }^{3}$ School of Electrical and Electronic Engineering, Nanyang Technological University, Singapore 639798
}

Correspondence should be addressed to Yong-Hui Xia; yhxia@zjnu.cn

Received 8 April 2014; Accepted 21 April 2014; Published 5 May 2014

Academic Editor: Jifeng Chu

Copyright (C) 2014 Yong-Hui Xia et al. This is an open access article distributed under the Creative Commons Attribution License, which permits unrestricted use, distribution, and reproduction in any medium, provided the original work is properly cited.

\begin{abstract}
Nonuniform exponential dichotomy has been investigated extensively. The essential condition of these previous results is based on the assumption that the nonlinear term satisfies $|f(t, x)| \leq \mu e^{-\varepsilon|t|}$. However, this condition is very restricted. There are few functions satisfying $|f(t, x)| \leq \mu e^{-\varepsilon|t|}$. In some sense, this assumption is not reasonable enough. More suitable assumption should be $|f(t, x)| \leq \mu$. To the best of the authors' knowledge, there is no paper considering the existence and uniqueness of solution to the perturbed nonautonomous system with a relatively conservative assumption $|f(t, x)| \leq \mu$. In this paper, we prove that if the nonlinear term is bounded, the perturbed nonautonomous system with nonuniform exponential dichotomy has a unique solution. The technique employed to prove Theorem 4 is the highlight of this paper.
\end{abstract}

\section{Introduction}

The notion of exponential dichotomy, introduced by Perron in [1], plays an important role in the theory of differential equations and dynamical systems (also see [2-5]). It is well known that if linear system $\dot{x}(t)=A(t) x(t)$ admits an (uniform) exponential dichotomy, the nonlinear term $f(t, x)$ is bounded and has a small Lipschitz constant, then the nonlinear system $\dot{x}(t)=A(t) x(t)+f(t, x)$ has a unique bounded solution (see [6]). However, many scholars argued that (uniform) exponential dichotomy restricted the behavior of dynamical systems. For this reason, we need a more general concept of hyperbolicity. Recently, Barreira and Valls $[7,8]$ have introduced the notion of nonuniform exponential dichotomy. General nonuniform exponential dichotomy has also been proposed (see [9-11]). Many properties of nonuniform exponential dichotomy have been extensively studied. For example, the topological conjugacies between linear and nonlinear perturbations were explored and some new Grobman-Hartman type theorems for nonuniform exponential dichotomy were established $([12,13])$. However, the essential condition of these results is based on the assumption that the nonlinear term satisfies $|f(t, x)| \leq \mu e^{-\varepsilon|t|}$. Under the same condition, Zhang et al. studied nonlinear perturbations of nonuniform exponential dichotomy on measure chains ([14]).

However, the condition $|f(t, x)| \leq \mu e^{-\varepsilon|t|}$ is very restricted. There are few functions satisfying $|f(t, x)| \leq$ $\mu e^{-\varepsilon|t|}$. Thus, it is necessary to find a more conservative condition for the nonlinear term $f(t, x)$. In this paper, our main objective is to explore the existence and uniqueness of solution to the perturbed nonautonomous system with a relatively conservative assumption $|f(t, x)| \leq \mu$. Finally, we prove that if $|f(t, x)| \leq \mu$, the perturbed nonautonomous system with nonuniform exponential dichotomy has a unique solution $x(t)$ satisfying $|x(t)|=O\left(e^{\varepsilon|t|}\right)$. 
The outline of this paper is arranged as follows. Next section is to state our main results. In Section 3, we prove the main results.

\section{Main Results}

In this section, we will state our main theorems. First, we introduce the definition of nonuniform exponential dichotomy.

Consider systems

$$
\begin{aligned}
& \dot{x}(t)=A(t) x(t), \\
& \dot{x}(t)=A(t) x(t)+f(t, x),
\end{aligned}
$$

where $t \in \mathbb{R}, x \in \mathbb{R}^{n}, A(t)$ is a continuous matrix function, and $f: \mathbb{R} \times \mathbb{R}^{n} \rightarrow \mathbb{R}^{n}$ is a continuous function.

Let $T_{A}(t, s)$ be the evolution operator satisfying $x(t)=$ $T_{A}(t, s) x(s), t, s \in \mathbb{R}$, where $x(t)$ is a solution of (1).

Definition 1. Linear system (1) is said to admit a nonuniform exponential dichotomy if there exists a projection $P(t)\left(P^{2}=\right.$ $P$ ) and constants $\alpha>0, K>0, \varepsilon \geq 0$, such that

$$
\begin{gathered}
\left|T_{A}(t, s) P(s)\right| \leq K e^{-\alpha(t-s)} \cdot e^{\varepsilon|s|}, \quad t \geq s, \\
\left|T_{A}(t, s) Q(s)\right| \leq K e^{\alpha(t-s)} \cdot e^{\varepsilon|s|}, \quad t \leq s,
\end{gathered}
$$

where $P(t)+Q(t)=$ Id (identity), $T_{A}(t, s) P(s)=$ $P(t) T_{A}(t, s), t, s \in \mathbb{R}$.

Remark 2. When $\varepsilon \equiv 0$, system (1) is said to have an exponential dichotomy; and when $\varepsilon \equiv 0, \alpha \equiv 0$, system (1) is said to have a uniform dichotomy.

To present our main results, we give a theorem under the trivial condition $|f(t, x)| \leq \mu e^{-\varepsilon|t|}$.

Theorem 3. Suppose that linear system (1) admits a nonuniform exponential dichotomy. For $t \in \mathbb{R}, x, x_{1}, x_{2} \in \mathbb{R}^{n}$, if the nonlinear term $f(t, x)$ satisfies

$$
\begin{aligned}
& \left(\widetilde{\mathrm{H}}_{1}\right)|f(t, x)| \leq \mu e^{-\varepsilon|t|}, \\
& \left(\widetilde{\mathrm{H}}_{2}\right)\left|f\left(t, x_{1}\right)-f\left(t, x_{2}\right)\right| \leq r e^{-\varepsilon|t|}\left|x_{1}-x_{2}\right|, \\
& \left(\widetilde{\mathrm{H}}_{3}\right) 4 K r<\alpha,
\end{aligned}
$$

where $\mu, r, \varepsilon, \alpha$ are all positive constants, then nonlinear system (2) has a unique bounded solution $\tilde{x}(t)$ satisfying

$$
\begin{aligned}
\tilde{x}(t)= & \int_{-\infty}^{t} T_{A}(t, s) P(s) f(s, \tilde{x}(s)) d s \\
& -\int_{t}^{+\infty} T_{A}(t, s) Q(s) f(s, \tilde{x}(s)) d s .
\end{aligned}
$$

Discussion. One of the essential conditions of Theorem 3 is $\left(\widetilde{\mathrm{H}}_{1}\right)$. However, this condition is very restricted. There are few functions satisfying $|f(t, x)| \leq \mu e^{-\varepsilon|t|}$. Thus, it is necessary to find a more conservative condition for the nonlinear term $f(t, x)$. The main objective of this paper is to prove that the perturbed system has a unique solution under $|f(t, x)| \leq \mu$. But Theorem 3 cannot be valid yet. For this case, we have the following.

Theorem 4. Suppose that linear system (1) admits a nonuniform exponential dichotomy with the estimates (3). For $t \in$ $\mathbb{R}, x, x_{1}, x_{2} \in \mathbb{R}^{n}$, if $f(t, x)$ satisfies

$$
\begin{aligned}
& \left(\mathrm{H}_{1}\right)|f(t, x)| \leq \mu, \\
& \left(\mathrm{H}_{2}\right)\left|f\left(t, x_{1}\right)-f\left(t, x_{2}\right)\right| \leq r e^{-\varepsilon|t|}\left|x_{1}-x_{2}\right|, \\
& \left(\mathrm{H}_{3}\right) 4 K r<\alpha-\varepsilon
\end{aligned}
$$

where $\alpha-\varepsilon$ is a positive constant, then system (2) has a unique solution $x(t)$ satisfying

$$
|x(t)|=O\left(e^{\varepsilon|t|}\right) .
$$

Remark 5. The method used to prove Theorem 3 cannot be applied to this case. To see how to overcome the difficulty, one can refer to the main proof of Theorem 4 . The technique employed to prove Theorem 4 is very skillful and interesting, which is the highlight of this paper.

\section{Proofs of Main Results}

In what follows, to prove Theorem 3, a preliminary lemma is needed.

Lemma 6 (see [15], Lemma 4). If system (1) admits a nonuniform exponential dichotomy, then system (1) has no nontrivial bounded solutions; that is, $x(t)=0$ is the unique bounded solution of (1).

3.1. Proof of Theorem 3. Let $\mathbf{B}=\{\varphi(t) \mid \varphi(t)$ be continuous and $\left.|\varphi(t)| \leq 2 K \mu \alpha^{-1}\right\}$, for $\forall \varphi \in \mathbf{B}$, define a mapping $\mathscr{T}_{1}$ :

$$
\begin{aligned}
\mathscr{T}_{1} \varphi(t)= & \int_{-\infty}^{t} T_{A}(t, s) P(s) f(s, \varphi(s)) d s \\
& -\int_{t}^{+\infty} T_{A}(t, s) Q(s) f(s, \varphi(s)) d s .
\end{aligned}
$$

From $(3)$ and $\left(\widetilde{\mathrm{H}}_{1}\right)$ and $\left(\widetilde{\mathrm{H}}_{2}\right)$, we have

$$
\begin{aligned}
\left|\mathscr{T}_{1} \varphi(t)\right| \leq & \int_{-\infty}^{t} K e^{-\alpha(t-s)} e^{\varepsilon|s|} \cdot \mu e^{-\varepsilon|s|} d s \\
& +\int_{t}^{+\infty} K e^{\alpha(t-s)} e^{\varepsilon|s|} \cdot \mu e^{-\varepsilon|s|} d s \\
= & K \mu \alpha^{-1}+K \mu \alpha^{-1} \\
= & 2 K \mu \alpha^{-1} .
\end{aligned}
$$


Therefore, $\mathscr{T}_{1} \varphi(t) \in \mathbf{B}$, which implies $\mathscr{T}_{1}$ maps $\mathbf{B}$ onto itself. On the other hand,

$$
\begin{aligned}
\left|\mathscr{T}_{1} \varphi_{1}(t)-\mathscr{T}_{1} \varphi_{2}(t)\right| & \\
\leq & \int_{-\infty}^{t} T_{A}(t, s) P(s) r e^{-\varepsilon|s|}\left|\varphi_{1}(s)-\varphi_{2}(s)\right| d s \\
& +\int_{t}^{+\infty} T_{A}(t, s) Q(s) r e^{-\varepsilon|s|}\left|\varphi_{1}(s)-\varphi_{2}(s)\right| d s \\
\leq & \int_{-\infty}^{t} K r e^{-\alpha(t-s)}\left|\varphi_{1}(s)-\varphi_{2}(s)\right| d s \\
& +\int_{t}^{+\infty} K r e^{\alpha(t-s)}\left|\varphi_{1}(s)-\varphi_{2}(s)\right| d s \\
\leq & 2 K r \alpha^{-1} \sup _{s \geq 0}\left|\varphi_{1}(s)-\varphi_{2}(s)\right| \\
\leq & \frac{1}{2} \sup _{s \geq 0}\left|\varphi_{1}(s)-\varphi_{2}(s)\right| .
\end{aligned}
$$

Then $\mathscr{T}_{1}$ is a contraction mapping. Therefore, in $\mathbf{B}$, there exists a unique fixed point $\varphi_{0}(t)$, such that

$$
\begin{aligned}
\varphi_{0}(t)=\mathscr{T}_{1} \varphi_{0}(t)= & \int_{-\infty}^{t} T_{A}(t, s) P(s) f\left(s, \varphi_{0}(s)\right) d s \\
& -\int_{t}^{+\infty} T_{A}(t, s) Q(s) f\left(s, \varphi_{0}(s)\right) d s
\end{aligned}
$$

Differentiating the above equality, we see that $\varphi_{0}(t)$ satisfies (2). Now we are going to show that the solution of system (2) satisfying $\left(\widetilde{\mathrm{H}}_{1}\right),\left(\widetilde{\mathrm{H}}_{2}\right)$, and $\left(\widetilde{\mathrm{H}}_{3}\right)$ is unique. Assume that system (2) has another bounded solution $\varphi^{*}(t)$ satisfying $\left(\widetilde{\mathrm{H}}_{1}\right),\left(\widetilde{\mathrm{H}}_{2}\right)$, and $\left(\widetilde{\mathrm{H}}_{3}\right)$; we have

$$
\begin{aligned}
\varphi^{*}(t)= & T_{A}(t, 0) \varphi^{*}(0) \\
& +\int_{0}^{t} T_{A}(t, s) T^{-1}(s, s) f\left(s, \varphi^{*}(s)\right) d s \\
= & T_{A}(t, 0) \varphi^{*}(0)+\int_{0}^{t} T_{A}(t, s) P(s) f\left(s, \varphi^{*}(s)\right) d s \\
& +\int_{0}^{t} T_{A}(t, s) Q(s) f\left(s, \varphi^{*}(s)\right) d s \\
= & T_{A}(t, 0) \varphi^{*}(0)+\int_{-\infty}^{t} T_{A}(t, s) P(s) f\left(s, \varphi^{*}(s)\right) d s
\end{aligned}
$$

$$
\begin{aligned}
& -\int_{-\infty}^{0} T_{A}(t, s) P(s) f\left(s, \varphi^{*}(s)\right) d s \\
& +\int_{0}^{+\infty} T_{A}(t, s) Q(s) f\left(s, \varphi^{*}(s)\right) d s \\
& -\int_{t}^{+\infty} T_{A}(t, s) Q(s) f\left(s, \varphi^{*}(s)\right) d s \\
& =T_{A}(t, 0)\left[\varphi^{*}(0)\right. \\
& \quad-\left(\int_{-\infty}^{0} T_{A}(0, s) P(s) f\left(s, \varphi^{*}(s)\right) d s\right. \\
& +\left(\int_{-\infty}^{t} T_{A}(t, s) P(s) f\left(s, \varphi^{*}(s)\right) d s\right. \\
& \left.-\int_{t}^{+\infty} T_{A}(t, s) Q(s) f\left(s, \varphi^{*}(s)\right) d s\right) .
\end{aligned}
$$

By calculating, we get

$$
\begin{aligned}
& \int_{-\infty}^{t} T_{A}(t, s) P(s) f\left(s, \varphi^{*}(s)\right) d s \\
& -\int_{t}^{+\infty} T_{A}(t, s) Q(s) f\left(s, \varphi^{*}(s)\right) d s \leq 2 K \mu \alpha^{-1} .
\end{aligned}
$$

As $\varphi^{*}(t)$ is bounded, we obtain

$$
\begin{aligned}
T_{A}(t, 0)\left[\varphi^{*}(0)-\right. & \left(\int_{-\infty}^{0} T_{A}(0, s) P(s) f\left(s, \varphi^{*}(s)\right) d s\right. \\
& \left.\left.-\int_{0}^{+\infty} T_{A}(0, s) Q(s) f\left(s, \varphi^{*}(s)\right) d s\right)\right],
\end{aligned}
$$

is bounded. In addition, the formula above is the solution of system (1), so it is a bounded solution. From Lemma 6, we have

$$
\begin{aligned}
T_{A}(t, 0) & {\left[\varphi^{*}(0)\right.} \\
& -\left(\int_{-\infty}^{0} T_{A}(0, s) P(s) f\left(s, \varphi^{*}(s)\right) d s\right. \\
& \left.\left.\quad-\int_{0}^{+\infty} T_{A}(0, s) Q(s) f\left(s, \varphi^{*}(s)\right) d s\right)\right]=0 .
\end{aligned}
$$

Therefore,

$$
\begin{aligned}
\varphi^{*}(t)= & \int_{-\infty}^{t} T_{A}(t, s) P(s) f\left(s, \varphi^{*}(s)\right) d s \\
& -\int_{t}^{+\infty} T_{A}(t, s) Q(s) f\left(s, \varphi^{*}(s)\right) d s .
\end{aligned}
$$


From (3), $\left(\widetilde{\mathrm{H}}_{2}\right)$, and $\left(\widetilde{\mathrm{H}}_{3}\right)$, we have

$$
\begin{aligned}
\left|\varphi_{0}(t)-\varphi^{*}(t)\right| & \\
\leq & \int_{-\infty}^{t} K e^{-\alpha(t-s)} e^{\varepsilon|s|} \cdot r e^{-\varepsilon|s|}\left|\varphi_{0}(s)-\varphi^{*}(s)\right| d s \\
& \quad+\int_{t}^{+\infty} K e^{\alpha(t-s)} e^{\varepsilon|s|} \cdot r e^{-\varepsilon|s|}\left|\varphi_{0}(s)-\varphi^{*}(s)\right| d s \\
= & 2 K r \alpha^{-1}\left|\varphi_{0}(s)-\varphi^{*}(s)\right| \\
= & \frac{1}{2} \sup _{t \in \mathbb{R}}\left|\varphi_{0}(t)-\varphi^{*}(t)\right| .
\end{aligned}
$$

That is, $\sup _{t \in \mathbb{R}}\left|\varphi_{0}(t)-\varphi^{*}(t)\right| \leq(1 / 2) \sup _{t \in \mathbb{R}}\left|\varphi_{0}(t)-\varphi^{*}(t)\right|$, which implies $\varphi_{0}(t)=\varphi^{*}(t)$. Then the uniqueness is proved. The proof of Theorem 3 is complete.

3.2. Proof of Theorem 4. To prove Theorem 4, a standard method is to employ a linear transformation $x=e^{\varepsilon|t|} y$. However, $x=e^{\varepsilon|t|} y$ is not differentiable at $t=0$. Thus, we cannot use such transformation directly. We have to discuss by dividing into two pieces $t \geq 0$ and $t \leq 0$.

Consider system

$$
\dot{x}(t)=B(t) v(t)+F(t, v),
$$

where $u \in \mathbb{R}^{n}, B(t)$ is a continuous matrix function, and $F$ : $\mathbb{R} \times \mathbb{R}^{n} \rightarrow \mathbb{R}^{n}$ is a continuous function.

Lemma 7. Suppose that system $\dot{v}(t)=B(t) v(t)$ admits a nonuniform exponential dichotomy; that is, its evolution operator $T_{B}(t, s)$ satisfies

$$
\begin{aligned}
& \left|T_{B}(t, s) P(s)\right| \leq K e^{-\lambda(t-s)} \cdot e^{\varepsilon|s|}, \quad t \geq s, \\
& \left|T_{B}(t, s) Q(s)\right| \leq K e^{\lambda(t-s)} \cdot e^{\varepsilon|s|}, \quad t \leq s,
\end{aligned}
$$

where $\lambda$ is a positive constant. In addition,

$$
\begin{gathered}
\left|F\left(t, v_{1}\right)-F\left(t, v_{2}\right)\right| \leq r e^{-\varepsilon|t|}\left|v_{1}-v_{2}\right|, \\
4 K r<\lambda .
\end{gathered}
$$

If $|F(t, v)| \leq \mu e^{-\varepsilon|t|}$ for $t \geq 0$, then for any $a \in \mathbb{R}^{n}$, system (16) has a unique solution $v^{+}(t)$ satisfying the following:

(i) $\left|v^{+}(t)\right|<+\infty$, for $t \geq 0$;

(ii) $P(0) v^{+}(0)=P(0) a$;

(iii) in $\mathbb{R}^{+}, v^{+}(t)$ satisfies integral equation

$$
\begin{aligned}
v^{+}(t)= & T_{B}(t, 0) P(0) a+\int_{0}^{t} T_{B}(t, s) P(s) F\left(s, v^{+}(s)\right) d s \\
& -\int_{t}^{+\infty} T_{B}(t, s) Q(s) F\left(s, v^{+}(s)\right) d s .
\end{aligned}
$$

If $|F(t, v)| \leq \mu e^{-\varepsilon|t|}$ for $t \leq 0$, then for any $a \in \mathbb{R}^{n}$, system (16) has a unique solution $v^{-}(t)$ satisfying the following: (i) $\left|v^{-}(t)\right|<+\infty$, for $t \leq 0$;

(ii) $Q(0) v^{-}(0)=Q(0) a$;

(iii) in $\mathbb{R}^{-}, v^{-}(t)$ satisfies integral equation

$$
\begin{aligned}
v^{-}(t)= & T_{B}(t, 0) Q(0) a+\int_{-\infty}^{t} T_{B}(t, s) P(s) F\left(s, v^{-}(s)\right) d s \\
& -\int_{t}^{0} T_{B}(t, s) Q(s) F\left(s, v^{-}(s)\right) d s .
\end{aligned}
$$

Proof. We prove the existence of $v^{+}(t)$ by successive approximation method. For any $a \in \mathbb{R}^{n}$, let $v_{0}^{+}(t)=T_{B}(t, 0) P(0) a$. We define $v_{m}^{+}(t), v_{m+1}^{+}(t)$ recursively as follows:

$$
\begin{aligned}
v_{m+1}^{+}(t)= & T_{B}(t, 0) P(0) a+\int_{0}^{t} T_{B}(t, s) P(s) F\left(s, v_{m}^{+}(s)\right) d s \\
& -\int_{t}^{+\infty} T_{B}(t, s) Q(s) F\left(s, v_{m}^{+}(s)\right) d s .
\end{aligned}
$$

From (17) and $|F(t, v)| \leq \mu e^{-\varepsilon|t|}$, for $t \geq 0$, we have

$$
\begin{aligned}
\left|v_{m+1}^{+}(t)\right| \leq & K e^{-\lambda t}|a|+\int_{0}^{t} K e^{-\lambda(t-s)} e^{\varepsilon s} \cdot \mu e^{-\varepsilon s} d s \\
& +\int_{t}^{+\infty} K e^{\lambda(t-s)} e^{\varepsilon s} \cdot \mu e^{-\varepsilon s} d s \\
= & K e^{-\lambda t}|a|+K \mu \lambda^{-1}\left(1-e^{-\lambda t}\right)+K \mu \lambda^{-1} \\
\leq & K|a|+2 K \mu \lambda^{-1} .
\end{aligned}
$$

For any bounded function $v(t)$ defined on $\mathbb{R}^{+}$, denote $\|v\|=$ $\sup _{t \in \mathbb{R}}|v(t)|$; then it follows from (18) and (21) that

$$
\begin{aligned}
& \left|v_{m+1}^{+}(t)-v_{m}^{+}(t)\right| \\
& \leq \int_{0}^{t} K e^{-\lambda(t-s)} e^{\varepsilon s} \cdot r e^{-\varepsilon s}\left|v_{m}^{+}(s)-v_{m-1}^{+}(s)\right| d s \\
& \quad+\int_{t}^{+\infty} K e^{\lambda(t-s)} e^{\varepsilon s} \cdot r e^{-\varepsilon s}\left|v_{m}^{+}(s)-v_{m-1}^{+}(s)\right| d s \\
& \leq 2 K r \lambda^{-1}\left|v_{m}^{+}(s)-v_{m-1}^{+}(s)\right| \\
& \leq 2 K r \lambda^{-1}\left\|v_{m}^{+}-v_{m-1}^{+}\right\| \\
& \leq \frac{1}{2}\left\|v_{m}^{+}-v_{m-1}^{+}\right\|,
\end{aligned}
$$

which implies that $\left\|v_{m+1}^{+}-v_{m}^{+}\right\| \leq(1 / 2)\left\|v_{m}^{+}-v_{m-1}^{+}\right\|$. Hence, the series $\sum_{m=0}^{\infty}\left(v_{m+1}^{+}(t)-v_{m}^{+}(t)\right)$ converges uniformly on $\mathbb{R}^{+}$. It means that the series $\left\{v_{m}^{+}(t)\right\}$ converges uniformly to a limit $v^{+}(t)$ on $\mathbb{R}^{+}$.

From (21), for any fixed $t$, let $m \rightarrow \infty$, we have

$$
\begin{aligned}
v^{+}(t)= & T_{B}(t, 0) P(0) a+\int_{0}^{t} T_{B}(t, s) P(s) F\left(s, v^{+}(s)\right) d s \\
& -\int_{t}^{+\infty} T_{B}(t, s) Q(s) F\left(s, v^{+}(s)\right) d s .
\end{aligned}
$$


Differentiating the above equality, we see that $v^{+}(t)$ satisfies the system (16).

From (22) and (24), we know that $\left|v^{+}(t)\right|<\infty$ for $t \geq 0$, and it is easy to demonstrate that $P(0) v^{+}(0)=P(0) a$. Now we are going to show the uniqueness of $v^{+}(t)$. If there is another bounded function $\widetilde{v}^{+}(t)$ satisfying (i), (ii), and (iii) on $\mathbb{R}^{+}$, in view of (iii) and (18) we have

$$
\begin{aligned}
& \left|\widetilde{v}^{+}(t)-v^{+}(t)\right| \\
& \leq \int_{0}^{t} K e^{-\lambda(t-s)} e^{\varepsilon s} \cdot r e^{-\varepsilon s}\left|\widetilde{v}^{+}(s)-v^{+}(s)\right| d s \\
& \quad+\int_{t}^{+\infty} K e^{\lambda(t-s)} e^{\varepsilon s} \cdot r e^{-\varepsilon s}\left|\tilde{v}^{+}(s)-v^{+}(s)\right| d s \\
& \leq 2 K r \lambda^{-1}\left\|\tilde{v}^{+}-v^{+}\right\| \\
& \leq \frac{1}{2}\left\|\tilde{v}^{+}-v^{+}\right\|,
\end{aligned}
$$

which implies $\left\|\widetilde{v}^{+}-v^{+}\right\| \leq(1 / 2)\left\|\widetilde{v}^{+}-v^{+}\right\|$. Hence, $\widetilde{v}^{+}(t) \equiv v^{+}(t)$.

The proof of the existence and uniqueness of $v^{-}(t)$ is similar to that of $v^{+}(t)$. The proof of Lemma 7 is complete.

Lemma 8. Suppose that $\alpha>0, \delta>0, C, L$, and $M$ are nonnegative constants and that $v(t)$ is a nonnegative bounded continuous function which satisfies two of the following inequalities:

$$
\begin{aligned}
v(t) \leq & C e^{-\alpha t}+L \int_{0}^{t} e^{-\alpha(t-s)} v(s) d s \\
& +M \int_{t}^{+\infty} e^{\delta(t-s)} v(s) d s, \quad(t \geq 0) \\
v(t) \leq & C e^{\alpha t}+L \int_{t}^{0} e^{\alpha(t-s)} v(s) d s \\
& +M \int_{-\infty}^{t} e^{-\delta(t-s)} v(s) d s, \quad(t \leq 0) .
\end{aligned}
$$

In addition, if $\gamma=L / \alpha+M / \delta<1$, then for $t \geq 0$ or $t \leq 0$, one has

$$
v(t) \leq(1-\gamma)^{-1} C e^{-\left[\alpha-(1-\gamma)^{-1} L\right]|t|} .
$$

Proof. The proof is straightforward by Lemma 6.2 of Chapter 3 in [6].

Lemma 9. For any a $\in \mathbb{R}^{n}$, system (2) has a unique solution $x^{+}(t)$ with the following properties:

(i) $\left|x^{+}(t) e^{-\varepsilon t}\right|<+\infty$, for $t \geq 0$;

(ii) $P(0) x^{+}(0)=P(0) a$;

(iii) $x^{+}(t)$ on $\mathbb{R}^{+}$satisfies integral equation

$$
\begin{aligned}
x^{+}(t)= & T_{A}(t, 0) P(0) a+\int_{0}^{t} T_{A}(t, s) P(s) f\left(s, x^{+}(s)\right) d s \\
& -\int_{t}^{+\infty} T_{A}(t, s) Q(s) f\left(s, x^{+}(s)\right) d s .
\end{aligned}
$$

Similarly, for any $a \in \mathbb{R}^{n}$, system (2) also has a unique solution $x^{-}(t)$ with the following properties:

(i) $\left|x^{-}(t) e^{\varepsilon t}\right|<+\infty$, for $t \leq 0$;

(ii) $Q(0) x^{-}(0)=Q(0) a$;

(iii) $x^{-}(t)$ on $\mathbb{R}^{-}$satisfies integral equation

$$
\begin{aligned}
x^{-}(t)= & T_{A}(t, 0) Q(0) a+\int_{-\infty}^{t} T_{A}(t, s) P(s) f\left(s, x^{-}(s)\right) d s \\
& -\int_{t}^{0} T_{A}(t, s) Q(s) f\left(s, x^{-}(s)\right) d s .
\end{aligned}
$$

Proof. We firstly prove the existence and uniqueness of $x^{+}(t)$. Let $v=x e^{-\varepsilon t}$, then system (2) becomes

$$
\dot{v}(t)=(A(t)-\varepsilon I) v(t)+e^{-\varepsilon t} f\left(t, v e^{\varepsilon t}\right) .
$$

Let $F(t, v)=e^{-\varepsilon t} f\left(t, v e^{\varepsilon t}\right)$. From $\left(\mathrm{H}_{1}\right)$ and $\left(\mathrm{H}_{2}\right)$, we have

$$
\begin{aligned}
&|F(t, v)| \leq e^{-\varepsilon t} \mu=\mu e^{-\varepsilon|t|}, \quad \text { for } t \geq 0 ; \\
&\left|F\left(t, v_{1}\right)-F\left(t, v_{2}\right)\right|=e^{-\varepsilon t}\left|f\left(t, v_{1} e^{\varepsilon t}\right)-f\left(t, v_{2} e^{\varepsilon t}\right)\right| \\
& \leq e^{-\varepsilon t} r e^{-\varepsilon|t|}\left|v_{1} e^{\varepsilon t}-v_{2} e^{\varepsilon t}\right| \\
& \leq r e^{-\varepsilon|t|}\left|v_{1}-v_{2}\right| .
\end{aligned}
$$

Let $T_{C}(t, s)$ be the evolution operator of the linear system $\dot{v}(t)=(A(t)-\varepsilon t) v(t)$. Since $x(t)=T_{A}(t, s) x(s), v=e^{-\varepsilon t} x$, we have $T_{C}(t, s)=e^{-\varepsilon(t-s)} T_{A}(t, s)$. Hence, from (3), we obtain

$$
\begin{gathered}
\left|T_{C}(t, s) P(s)\right| \leq K e^{-(\alpha-\varepsilon)(t-s)} \cdot e^{\varepsilon|s|}, \quad \text { for } t \geq s, \\
\left|T_{C}(t, s) Q(s)\right| \leq K e^{(\alpha-\varepsilon)(t-s)} \cdot e^{\varepsilon|s|}, \quad \text { for } t \leq s .
\end{gathered}
$$

Since $4 K r<\alpha-\varepsilon$, system (30) satisfies all conditions of Lemma 7. Therefore, for any $a \in \mathbb{R}^{n}$, system (30) has a unique solution $v^{+}(t)$ with the following properties:
(i) $\left|v^{+}(t)\right|<+\infty$, for $t \geq 0$;
(ii) $P(0) v^{+}(0)=P(0) a$;
(iii) $v^{+}(t)$ on $\mathbb{R}^{+}$satisfies integral equation

$$
\begin{aligned}
v^{+}(t)= & T_{C}(t, 0) P(0) a+\int_{0}^{t} T_{C}(t, s) P(s) F\left(s, v^{+}(s)\right) d s \\
& -\int_{t}^{+\infty} T_{C}(t, s) Q(s) F\left(s, v^{+}(s)\right) d s \\
= & e^{-\varepsilon t} T_{A}(t, 0) P(0) a \\
& +\int_{0}^{t} e^{-\varepsilon(t-s)} T_{A}(t, s) P(s) \cdot e^{-\varepsilon s} f\left(s, v^{+}(s) e^{\varepsilon s}\right) d s \\
& -\int_{t}^{+\infty} e^{-\varepsilon(t-s)} T_{A}(t, s) Q(s) \\
& \cdot e^{-\varepsilon s} f\left(s, v^{+}(s) e^{\varepsilon s}\right) d s .
\end{aligned}
$$


Hence,

$$
\begin{aligned}
v^{+}(t) e^{\varepsilon t}= & T_{A}(t, 0) P(0) a \\
& +\int_{0}^{t} T_{A}(t, s) P(s) f\left(s, v^{+}(s) e^{\varepsilon s}\right) d s \\
& -\int_{t}^{+\infty} T_{A}(t, s) Q(s) f\left(s, v^{+}(s) e^{\varepsilon s}\right) d s .
\end{aligned}
$$

Let $x^{+}(t)=v^{+}(t) e^{\varepsilon t}$, we have

$$
\begin{aligned}
x^{+}(t)= & T_{A}(t, 0) P(0) a+\int_{0}^{t} T_{A}(t, s) P(s) f\left(s, x^{+}(s)\right) d s \\
& -\int_{t}^{+\infty} T_{A}(t, s) Q(s) f\left(s, x^{+}(s)\right) d s .
\end{aligned}
$$

Then $x^{+}(t)$ is the solution of system (2) and it satisfies all conditions of Lemma 9.

The proof for the existence and uniqueness of $x^{-}(t)$ is similar to that of $x^{+}(t)$, so we omit it. This completes the proof of Lemma 9.

Lemma 10. If $\left|T_{A}(t, 0) P(0) a\right| \leq M e^{\varepsilon|t|}$, then $a=0$.

Proof. If $a \neq 0$, then $P(0) a \neq 0$ or $Q(0) a \neq 0$. Without loss of generality, we assume $P(0) a \neq 0$, then we have

$$
\begin{aligned}
\left|T_{A}(t, 0) P(0) a\right| & =\left|T_{A}(t, 0) P(0) T_{A}^{-1}(s, 0) T_{A}(s, 0) P(0) a\right| \\
& =\left|T_{A}(t, 0) T_{A}(0, s) P(s) T_{A}(s, 0) P(0) a\right| \\
& =\left|T_{A}(t, s) P(s) T_{A}(s, 0) P(0) a\right| \\
& \leq\left|T_{A}(t, s) P(s)\right| \cdot\left|T_{A}(s, 0) P(0) a\right|
\end{aligned}
$$

Since $\left|T_{A}(t, s) P(s)\right| \leq K e^{-\alpha(t-s)} e^{\varepsilon|s|}$ for $t \geq s$,

$$
\left|T_{A}(t, 0) P(0) a\right| \leq K e^{-\alpha(t-s)} e^{\varepsilon|s|} \cdot\left|T_{A}(s, 0) P(0) a\right|,
$$

which implies

$$
\left|T_{A}(s, 0) P(0) a\right| \geq \frac{\left|T_{A}(t, 0) P(0) a\right|}{K e^{-\alpha(t-s)} e^{\varepsilon|s|}} .
$$

Taking $t=0$, we obtain

$$
\begin{aligned}
\left|T_{A}(s, 0) P(0) a\right| & \geq \frac{|P(0) a|}{K e^{\alpha s} e^{-\varepsilon s}} \\
& =K^{-1} e^{-(\alpha-\varepsilon) s}|P(0) a| \quad(s \leq 0) .
\end{aligned}
$$

Therefore, when $s \leq 0$,

$$
\frac{\left|T_{A}(s, 0) P(0) a\right|}{e^{\varepsilon|s|}} \geq K^{-1} e^{-\alpha s}|P(0) a| \longrightarrow+\infty \quad \text { as } s \longrightarrow-\infty \text {. }
$$

On the other hand, when $s \leq 0$, from (3), we have

$$
\left|T_{A}(s, 0) Q(0) a\right| \leq K e^{\alpha s} e^{\varepsilon|0|}=K e^{\alpha s},
$$

hence,

$$
\frac{\left|T_{A}(s, 0) Q(0) a\right|}{e^{\varepsilon|s|}} \leq K e^{(\alpha+\varepsilon) s}
$$

It follows from (40) and (42) that

$$
\begin{aligned}
\frac{\left|T_{A}(s, 0) a\right|}{e^{\varepsilon|s|}} & =\frac{\left|T_{A}(s, 0)(P(s)+Q(s)) a\right|}{e^{\varepsilon|s|}} \\
& \geq \frac{\left|T_{A}(s, 0) P(s) a\right|}{e^{\varepsilon|s|}}-\frac{\left|T_{A}(s, 0) Q(s) a\right|}{e^{\varepsilon|s|}} \\
& \geq \frac{\left|T_{A}(s, 0) P(s) a\right|}{e^{\varepsilon|s|}}-K e^{(\alpha+\varepsilon) s} .
\end{aligned}
$$

From the above inequality, we know that $\left|T_{A}(s, 0) a\right| / e^{\varepsilon|s|} \rightarrow$ $+\infty$ as $s \rightarrow-\infty$, which contradicts the original condition $\left|T_{A}(s, 0) a\right| / e^{\varepsilon|s|} \leq M$ and it implies $a=0$. This ends the proof of Lemma 10.

Proof of Theorem 4. For any solution $x(t)$ of system (2), it can be written as follows:

$$
\begin{aligned}
& x(t)=T_{A}(t, 0) x(0)+\int_{0}^{t} T_{A}(t, s) T_{A}^{-1}(s, s) f(s, x(s)) d s \\
& =T_{A}(t, 0) x(0)+\int_{0}^{t} T_{A}(t, s) P(s) f(s, x(s)) d s \\
& +\int_{0}^{t} T_{A}(t, s) Q(s) f(s, x(s)) d s \\
& =T_{A}(t, 0) x(0)+\int_{-\infty}^{t} T_{A}(t, s) P(s) f(s, x(s)) d s \\
& -\int_{-\infty}^{0} T_{A}(t, s) P(s) f(s, x(s)) d s \\
& +\int_{0}^{+\infty} T_{A}(t, s) Q(s) f(s, x(s)) d s \\
& -\int_{t}^{+\infty} T_{A}(t, s) Q(s) f(s, x(s)) d s \\
& =T_{A}(t, 0)[x(0) \\
& -\left(\int_{-\infty}^{0} T_{A}(0, s) P(s) f(s, x(s)) d s\right. \\
& \left.\left.-\int_{0}^{+\infty} T_{A}(0, s) Q(s) f(s, x(s)) d s\right)\right] \\
& +\left(\int_{-\infty}^{t} T_{A}(t, s) P(s) f(s, x(s)) d s\right. \\
& \left.-\int_{t}^{+\infty} T_{A}(t, s) Q(s) f(s, x(s)) d s\right) .
\end{aligned}
$$


Let $\xi(t)$ be any $n$-variable continuous function defined on $\mathbb{R}$. From (3) and $\left(\mathrm{H}_{1}\right)$, we have

$$
\begin{aligned}
\left|\int_{-\infty}^{t} T_{A}(t, s) P(s) f(s, \xi(s)) d s\right| & \leq \int_{-\infty}^{t} K e^{-\alpha(t-s)} e^{\varepsilon|s|} \mu d s \\
& =K \mu e^{-\alpha t} \int_{-\infty}^{t} e^{\alpha s} e^{\varepsilon|s|} d s .
\end{aligned}
$$

For $t \geq 0$,

$$
\begin{aligned}
e^{-\alpha t} \int_{-\infty}^{t} e^{\alpha s} e^{\varepsilon|s|} d s & =e^{-\alpha t}\left(\int_{-\infty}^{0} e^{\alpha s} e^{-\varepsilon s} d s+\int_{0}^{t} e^{\alpha s} e^{\varepsilon s} d s\right) \\
& =e^{-\alpha t}\left(\frac{1}{\alpha-\varepsilon}+\frac{1}{\alpha+\varepsilon}\left(e^{(\alpha+\varepsilon) t}-1\right)\right) \\
& \leq \frac{1}{\alpha+\varepsilon} e^{\varepsilon t}+\frac{1}{\alpha-\varepsilon} \\
& \leq \frac{1}{\alpha-\varepsilon} e^{\varepsilon|t|}+\frac{1}{\alpha-\varepsilon}
\end{aligned}
$$

for $t \leq 0$,

$$
\begin{aligned}
e^{-\alpha t} \int_{-\infty}^{t} e^{\alpha s} e^{\varepsilon|s|} d s & =e^{-\alpha t} \int_{-\infty}^{t} e^{\alpha s} e^{-\varepsilon s} d s \\
& =\frac{1}{\alpha-\varepsilon} e^{-\varepsilon t} \\
& \leq \frac{1}{\alpha-\varepsilon} e^{\varepsilon|t|}+\frac{1}{\alpha-\varepsilon}
\end{aligned}
$$

Hence, for any $t \in \mathbb{R}$, we have

$$
e^{-\alpha t} \int_{-\infty}^{t} e^{\alpha s} e^{\varepsilon|s|} d s \leq \frac{1}{\alpha-\varepsilon} e^{\varepsilon|t|}+\frac{1}{\alpha-\varepsilon} .
$$

Therefore, for any $t \in \mathbb{R}$, we have

$$
\left|\int_{-\infty}^{t} T_{A}(t, s) P(s) f(s, \xi(s)) d s\right| \leq \frac{K \mu}{\alpha-\varepsilon} e^{\varepsilon|t|}+\frac{K \mu}{\alpha-\varepsilon} .
$$

By the same calculation, for any $t \in \mathbb{R}$, we have

$$
\left|\int_{t}^{+\infty} T_{A}(t, s) Q(s) f(s, \xi(s)) d s\right| \leq \frac{K \mu}{\alpha-\varepsilon} e^{\varepsilon|t|}+\frac{K \mu}{\alpha-\varepsilon} .
$$

In Lemma $9, x^{+}(t)$ and $x^{-}(t)$ are uniquely determined by $a$; we denote them by $x^{+}(t, a)$ and $x^{-}(t, a)$, respectively. Let $\rho=(2 K \mu) /(\alpha-\varepsilon)$, denote by $G$ the closed sphere on $\mathbb{R}^{n}$ whose center is at the origin of the coordinate system and whose radius is $\rho$. For any $a \in G$, we define a mapping $\mathscr{T}_{2}: G \rightarrow \mathbb{R}^{n}$ as follows:

$$
\begin{aligned}
\mathscr{T}_{2} a= & \int_{-\infty}^{0} T_{A}(0, s) P(s) f\left(s, x^{-}(s, a)\right) d s \\
& -\int_{0}^{+\infty} T_{A}(0, s) Q(s) f\left(s, x^{+}(s, a)\right) d s .
\end{aligned}
$$

It follows from $(3)$ and $\left(\mathrm{H}_{1}\right)$ that

$$
\begin{aligned}
\left|\mathscr{T}_{2} a\right| & \leq \int_{-\infty}^{0} K e^{\alpha s} e^{\varepsilon|s|} \mu d s+\int_{0}^{+\infty} K e^{-\alpha s} e^{\varepsilon|s|} \mu d s \\
& =\frac{K \mu}{\alpha-\varepsilon}+\frac{K \mu}{\alpha-\varepsilon} \\
& =\frac{2 K \mu}{\alpha-\varepsilon}=\rho,
\end{aligned}
$$

which implies that $\mathscr{T}_{2}$ maps $G$ onto itself. Now we are going to show that $\mathscr{T}_{2}$ is continuous. For any $a_{1}, a_{2} \in G$, from (3) and $\left(\mathrm{H}_{2}\right)$, we have

$$
\begin{aligned}
& \left|\mathscr{T}_{2} a_{1}-\mathscr{T}_{2} a_{2}\right| \\
& \leq \int_{-\infty}^{0} K e^{\alpha s} e^{\varepsilon|s|} \cdot r e^{-\varepsilon|s|}\left|x^{-}\left(s, a_{1}\right)-x^{-}\left(s, a_{2}\right)\right| d s \\
& \quad+\int_{0}^{+\infty} K e^{-\alpha s} e^{\varepsilon|s|} \cdot r e^{-\varepsilon|s|}\left|x^{+}\left(s, a_{1}\right)-x^{+}\left(s, a_{2}\right)\right| d s \\
& \leq \int_{-\infty}^{0} K r e^{\alpha s}\left|x^{-}\left(s, a_{1}\right)-x^{-}\left(s, a_{2}\right)\right| d s \\
& \quad+\int_{0}^{+\infty} K r e^{-\alpha s}\left|x^{+}\left(s, a_{1}\right)-x^{+}\left(s, a_{2}\right)\right| d s .
\end{aligned}
$$

From (3) and the condition (iii) of Lemma 9, for $t \geq 0$, we have

$$
\begin{aligned}
\mid x^{+}( & \left.t, a_{1}\right)-x^{+}\left(t, a_{2}\right) \mid \\
\leq & T_{A}(t, 0) P(0)\left|a_{1}-a_{2}\right| \\
& +\int_{0}^{t} K e^{-\alpha(t-s)} e^{\varepsilon|s|} \cdot r e^{-\varepsilon|s|}\left|x^{+}\left(s, a_{1}\right)-x^{+}\left(s, a_{2}\right)\right| d s \\
& +\int_{t}^{+\infty} K e^{\alpha(t-s)} e^{\varepsilon|s|} \cdot r e^{-\varepsilon|s|}\left|x^{+}\left(s, a_{1}\right)-x^{+}\left(s, a_{2}\right)\right| d s \\
\leq & K e^{-\alpha t}\left|a_{1}-a_{2}\right| \\
& +\int_{0}^{t} K r e^{-\alpha(t-s)}\left|x^{+}\left(s, a_{1}\right)-x^{+}\left(s, a_{2}\right)\right| d s \\
& +\int_{t}^{+\infty} K r e^{\alpha(t-s)}\left|x^{+}\left(s, a_{1}\right)-x^{+}\left(s, a_{2}\right)\right| d s .
\end{aligned}
$$

Multiplying by $e^{-\varepsilon t}$ on both sides of the above inequality, for $t \geq 0$, we get

$$
\begin{aligned}
& e^{-\varepsilon t}\left|x^{+}\left(t, a_{1}\right)-x^{+}\left(t, a_{2}\right)\right| \\
& \leq K e^{-(\alpha+\varepsilon) t}\left|a_{1}-a_{2}\right| \\
&+\int_{0}^{t} K r e^{-(\alpha+\varepsilon)(t-s)}\left(e^{-\varepsilon s}\left|x^{+}\left(s, a_{1}\right)-x^{+}\left(s, a_{2}\right)\right|\right) d s \\
&+\int_{t}^{+\infty} K r e^{(\alpha-\varepsilon)(t-s)}\left(e^{-\varepsilon s}\left|x^{+}\left(s, a_{1}\right)-x^{+}\left(s, a_{2}\right)\right|\right) d s
\end{aligned}
$$


By Lemma 9 , for $t \geq 0, e^{-\varepsilon t}\left|x^{+}\left(t, a_{1}\right)-x^{+}\left(t, a_{2}\right)\right|$ is a bounded function. And it follows from Lemma 8 that

$$
\begin{aligned}
& e^{-\varepsilon t}\left|x^{+}\left(t, a_{1}\right)-x^{+}\left(t, a_{2}\right)\right| \\
& \quad \leq K\left|a_{1}-a_{2}\right|(1-\gamma)^{-1} e^{-\left[(\alpha+\varepsilon)-(1-\gamma)^{-1} K r\right] t},
\end{aligned}
$$

where $\gamma=K r /(\alpha+\varepsilon)+K r /(\alpha-\varepsilon)$. From $\left(\mathrm{H}_{3}\right)$ and $(\alpha+\varepsilon)^{-1}<$ $(\alpha-\varepsilon)^{-1}$, we get

$$
\gamma \leq 2 K r(\alpha-\varepsilon)^{-1}<\frac{1}{2} .
$$

Therefore, for $\alpha-2 K r>0$, we have

$$
\begin{aligned}
e^{-\varepsilon t} & \left|x^{+}\left(t, a_{1}\right)-x^{+}\left(t, a_{2}\right)\right| \\
& \leq 2 K\left|a_{1}-a_{2}\right| e^{-[(\alpha+\varepsilon)-2 K r] t} \quad(t \geq 0) .
\end{aligned}
$$

Hence,

$$
\begin{aligned}
& \left|x^{+}\left(t, a_{1}\right)-x^{+}\left(t, a_{2}\right)\right| \\
& \quad \leq 2 K\left|a_{1}-a_{2}\right| e^{-(\alpha-2 K r) t} \quad(t \geq 0) .
\end{aligned}
$$

Similarly,

$$
\begin{aligned}
& \left|x^{-}\left(t, a_{1}\right)-x^{-}\left(t, a_{2}\right)\right| \\
& \quad \leq 2 K\left|a_{1}-a_{2}\right| e^{(\alpha-2 K r) t} \quad(t \leq 0) .
\end{aligned}
$$

So from (53), it follows that

$$
\begin{aligned}
\left|\mathscr{T}_{2} a_{1}-\mathscr{T}_{2} a_{2}\right| \leq & \int_{-\infty}^{0} K r e^{\alpha s} \cdot 2 K\left|a_{1}-a_{2}\right| e^{(\alpha-2 K r) s} d s \\
& +\int_{0}^{+\infty} K r e^{-\alpha s} \cdot 2 K\left|a_{1}-a_{2}\right| e^{-(\alpha-2 K r) s} d s \\
\leq & \int_{-\infty}^{0} 2 K^{2} r\left|a_{1}-a_{2}\right| e^{2(\alpha-K r) s} d s \\
& +\int_{0}^{+\infty} 2 K^{2} r\left|a_{1}-a_{2}\right| e^{-2(\alpha-K r) s} d s \\
= & \frac{2 K^{2} r}{\alpha-K r}\left|a_{1}-a_{2}\right|
\end{aligned}
$$

which show that $\mathscr{T}_{2}$ is a continuous mapping. By fixed point theorem, $\mathscr{T}_{2}$ has at least one fixed point on $G$. We denote this fixed point by $a_{0}$, then

$$
\begin{aligned}
a_{0}=\mathscr{T}_{2} a_{0}= & \int_{-\infty}^{0} T_{A}(0, s) P(s) f\left(s, x^{-}\left(s, a_{0}\right)\right) d s \\
& -\int_{0}^{+\infty} T_{A}(0, s) Q(s) f\left(s, x^{+}\left(s, a_{0}\right)\right) d s .
\end{aligned}
$$

As $P^{2}(s)=P(s), P(t) T_{A}(t, s)=T_{A}(t, s) P(s), P(s)+Q(s)=\mathrm{Id}$, we obtain

$$
\begin{gathered}
P(0) a_{0}=\int_{-\infty}^{0} T_{A}(0, s) P(s) f\left(s, x^{-}\left(s, a_{0}\right)\right) d s, \\
Q(0) a_{0}=-\int_{0}^{+\infty} T_{A}(0, s) Q(s) f\left(s, x^{-}\left(s, a_{0}\right)\right) d s .
\end{gathered}
$$

From Lemma 9, we have

$$
\begin{aligned}
& x^{+}\left(0, a_{0}\right)=P(0) a_{0}-\int_{0}^{+\infty} T_{A}(0, s) Q(s) f\left(s, x^{+}\left(s, a_{0}\right)\right) d s \\
& x^{-}\left(0, a_{0}\right)=Q(0) a_{0}+\int_{-\infty}^{0} T_{A}(0, s) P(s) f\left(s, x^{-}\left(s, a_{0}\right)\right) d s .
\end{aligned}
$$

Hence,

$$
x^{+}\left(0, a_{0}\right)=x^{-}\left(0, a_{0}\right)=a_{0} .
$$

By the existence and uniqueness of the initial value problem, we conclude that $x^{+}\left(t, a_{0}\right)=x^{-}\left(t, a_{0}\right)$. We can denote it by $x_{0}(t)$. Hence,

$$
\begin{aligned}
x_{0}(0)= & a_{0} \\
= & \int_{-\infty}^{0} T_{A}(0, s) P(s) f\left(s, x\left(s, a_{0}\right)\right) d s \\
& -\int_{0}^{+\infty} T_{A}(0, s) Q(s) f\left(s, x\left(s, a_{0}\right)\right) d s .
\end{aligned}
$$

From the above equation, it follows from (44) that

$$
\begin{aligned}
x_{0}(t)= & \int_{-\infty}^{t} T_{A}(t, s) P(s) f(s, x(s)) d s \\
& -\int_{t}^{+\infty} T_{A}(t, s) Q(s) f(s, x(s)) d s .
\end{aligned}
$$

From (49) and (50), we have

$$
\left|x_{0}(t)\right| \leq \frac{2 K \mu}{\alpha-\varepsilon} e^{\varepsilon|t|}+\frac{2 K \mu}{\alpha-\varepsilon}, \quad(-\infty<t<+\infty),
$$

which implies that $x_{0}(t)$ satisfies (5); that is, $x_{0}(t)=O\left(e^{\varepsilon|t|}\right)$.

Now we are going to prove that the solution of (2) which satisfies (5) is unique. We assume that system (2) has another solution $x_{0}^{*}(t)$ satisfying (5). From $(44), x_{0}^{*}(t)$ can be written as

$$
\begin{aligned}
& x_{0}^{*}(t)=T_{A}(t, 0)\left[x_{0}^{*}(0)\right. \\
&-\left(\int_{-\infty}^{0} T_{A}(0, s) P(s) f\left(s, x_{0}^{*}(s)\right) d s\right. \\
&\left.\left.-\int_{0}^{+\infty} T_{A}(0, s) Q(s) f\left(s, x_{0}^{*}(s)\right) d s\right)\right] \\
&+\left(\int_{-\infty}^{t} T_{A}(t, s) P(s) f\left(s, x_{0}^{*}(s)\right) d s\right. \\
&\left.-\int_{t}^{+\infty} T_{A}(t, s) Q(s) f\left(s, x_{0}^{*}(s)\right) d s\right) .
\end{aligned}
$$


From (49) and (50), we get

$$
\begin{aligned}
& \left|\int_{-\infty}^{t} T_{A}(t, s) P(s) f\left(s, x_{0}^{*}(s)\right) d s\right| \leq \frac{K \mu}{\alpha-\varepsilon} e^{\varepsilon|t|}+\frac{K \mu}{\alpha-\varepsilon}, \\
& \left|\int_{t}^{+\infty} T_{A}(t, s) Q(s) f\left(s, x_{0}^{*}(s)\right) d s\right| \leq \frac{K \mu}{\alpha-\varepsilon} e^{\varepsilon|t|}+\frac{K \mu}{\alpha-\varepsilon} .
\end{aligned}
$$

It follows from $\left|x_{0}^{*}(t)\right|=O\left(e^{\varepsilon|t|}\right)$ and Lemma 10 that

$$
\begin{aligned}
& x_{0}^{*}-\left(\int_{-\infty}^{0} T_{A}(0, s) P(s) f\left(s, x_{0}^{*}(s)\right) d s\right. \\
& \left.\quad-\int_{0}^{+\infty} T_{A}(0, s) Q(s) f\left(s, x_{0}^{*}(s)\right) d s\right)=0 .
\end{aligned}
$$

Therefore,

$$
\begin{aligned}
x_{0}^{*}(t)=\int_{-\infty}^{t} T_{A}(t, s) P(s) f\left(s, x_{0}^{*}(s)\right) d s \\
\quad-\int_{t}^{+\infty} T_{A}(t, s) Q(s) f\left(s, x_{0}^{*}(s)\right) d s .
\end{aligned}
$$

From (67), (72), and $\left(\mathrm{H}_{2}\right)$, we have

$$
\begin{aligned}
\left|x_{0}(t)-x_{0}^{*}(t)\right| & \\
\leq & \int_{-\infty}^{t} K e^{-\alpha(t-s)} e^{\varepsilon|s|} \cdot r e^{-\varepsilon|t|}\left|x_{0}(s)-x_{0}^{*}(s)\right| d s \\
& +\int_{t}^{+\infty} K e^{\alpha(t-s)} e^{\varepsilon|t|} \cdot r e^{-\varepsilon|t|}\left|x_{0}(s)-x_{0}^{*}(s)\right| d s \\
= & \int_{-\infty}^{t} K r e^{-\alpha(t-s)}\left|x_{0}(s)-x_{0}^{*}(s)\right| d s \\
& +\int_{t}^{+\infty} K r e^{\alpha(t-s)}\left|x_{0}(s)-x_{0}^{*}(s)\right| d s .
\end{aligned}
$$

Let $L=\sup _{t \in \mathbb{R}} e^{-\varepsilon|t|} \mid x_{0}(t)-x_{0}^{*}\left(t\right.$. Since $x_{0}(t)$ and $x_{0}^{*}(t)$ satisfy (5), $e^{-\varepsilon|s|}\left|x_{0}(s)-x_{0}^{*}(s)\right|$ is bounded. Thus, for $t \geq 0$, we have

$$
\begin{aligned}
& e^{-\varepsilon|t|}\left|x_{0}(t)-x_{0}^{*}(t)\right| \\
& =e^{-\varepsilon t}\left|x_{0}(t)-x_{0}^{*}(t)\right|
\end{aligned}
$$

$$
\begin{aligned}
\leq & \int_{-\infty}^{t} K e^{-(\alpha+\varepsilon)(t-s)} r\left[e^{-\varepsilon s}\left|x_{0}(s)-x_{0}^{*}(s)\right|\right] d s \\
& +\int_{t}^{+\infty} K e^{(\alpha-\varepsilon)(t-s)} r\left[e^{-\varepsilon s}\left|x_{0}(s)-x_{0}^{*}(s)\right|\right] d s \\
= & \int_{-\infty}^{0} K e^{-(\alpha+\varepsilon)(t-s)} r\left[e^{-\varepsilon s}\left|x_{0}(s)-x_{0}^{*}(s)\right|\right] d s \\
& +\int_{0}^{t} K e^{-(\alpha+\varepsilon)(t-s)} r\left[e^{-\varepsilon s}\left|x_{0}(s)-x_{0}^{*}(s)\right|\right] d s \\
& +\int_{t}^{+\infty} K e^{(\alpha-\varepsilon)(t-s)} r\left[e^{-\varepsilon s}\left|x_{0}(s)-x_{0}^{*}(s)\right|\right] d s \\
\leq & L\left(\frac{K r}{\alpha+\varepsilon} e^{-(\alpha-\varepsilon) t}+\frac{K r}{\alpha+\varepsilon}\left(1-e^{-(\alpha+\varepsilon) t}\right)+\frac{K r}{\alpha-\varepsilon}\right) \\
\leq & L\left(\frac{K r}{\alpha+\varepsilon}+\frac{K r}{\alpha+\varepsilon}+\frac{K r}{\alpha-\varepsilon}\right) \\
\leq & L\left(\frac{3 K r}{\alpha-\varepsilon}\right) \\
\leq & \frac{3}{4} L \quad\left(\mathrm{by}\left(\mathrm{H}_{3}\right)\right) .
\end{aligned}
$$

Similarly, we can prove that

$$
e^{-\varepsilon|t|}\left|x_{0}(t)-x_{0}^{*}(t)\right| \leq \frac{3}{4} L, \quad \text { when } t \leq 0 .
$$

Therefore,

$$
L=\sup _{t \in \mathbb{R}} e^{-\varepsilon|t|}\left|x_{0}(t)-x_{0}^{*}(t)\right| \leq \frac{3}{4} L, \quad(-\infty<t+\infty) .
$$

That is, $L \leq(3 / 4) L$, which implies $L=0$. Consequently, $x_{0}(t)=x_{0}^{*}(t)$. This completes the proof of Theorem 4 .

\section{Conflict of Interests}

The authors declare that there is no conflict of interests regarding the publication of this paper.

\section{Acknowledgment}

Yong-Hui Xia was supported by the National Natural Science Foundation of China under Grant nos. 11271333 and 11171090. Kit Ian Kou was supported by the University of Macau nos. MYRG142 (Y3-L2)-FST11-KKI, MRG002/KKI/2013/FST, MYRG099 (Y1-L2)-FST13-KKI, MRG 012/KKI/2014/FST and the Macao Science and Technology Development Fund FDCT/094/2011/A, FDCT/099/2012/A3.

\section{References}

[1] O. Perron, "Die stabilitätsfrage bei differentialgleichungen," Mathematische Zeitschrift, vol. 32, no. 1, pp. 703-728, 1930.

[2] W. A. Coppel, Dichotomies in Stability Theory, Lecture Notes in Mathematics, Springer, Berlin, Germany, 1978. 
[3] Y. Xia, J. Cao, and M. Han, "A new analytical method for the linearization of dynamic equation on measure chains," Journal of Differential Equations, vol. 235, no. 2, pp. 527-543, 2007.

[4] Y.-H. Xia, X. Chen, and V. G. Romanovski, "On the linearization theorem of Fenner and Pinto," Journal of Mathematical Analysis and Applications, vol. 400, no. 2, pp. 439-451, 2013.

[5] Y.-H. Xia, J. Li, and P. J. Y. Wong, "On the topological classification of dynamic equations on time scales," Nonlinear Analysis: Real World Applications, vol. 14, no. 6, pp. 2231-2248, 2013.

[6] J. K. Hale, Ordinary Differential Equations, Wiley-Interscience, New York, NY, USA, 1969.

[7] L. Barreira and C. Valls, "Smoothness of invariant manifolds for nonautonomous equations," Communications in Mathematical Physics, vol. 259, no. 3, pp. 639-677, 2005.

[8] L. Barreira and C. Valls, "Stable manifolds for nonautonomous equations without exponential dichotomy," Journal of Differential Equations, vol. 221, no. 1, pp. 58-90, 2006.

[9] L. Barreira, J. Chu, and C. Valls, "Lyapunov functions for general nonuniform dichotomies," Milan Journal of Mathematics, vol. 81, no. 1, pp. 153-169, 2013.

[10] L. Barreira, J. Chu, and C. Valls, "Robustness of nonuniform dichotomies with different growth rates," São Paulo Journal of Mathematical Sciences, vol. 5, no. 2, pp. 203-231, 2011.

[11] J. Chu, "Robustness of nonuniform behavior for discrete dynamics," Bulletin des Sciences Mathématiques, vol. 137, no. 8, pp. 1031-1047, 2013.

[12] L. Barreira and C. Valls, Stability of Nonautonomous Differential Equations, vol. 1926 of Lecture Notes in Mathematics, Springer, Berlin, Germany, 2008.

[13] L. Barreira and C. Valls, "A Grobman-Hartman theorem for nonuniformly hyperbolic dynamics," Journal of Differential Equations, vol. 228, no. 1, pp. 285-310, 2006.

[14] J. Zhang, M. Fan, and X. Chang, "Nonlinear perturbations of nonuniform exponential dichotomy on measure chains," Nonlinear Analysis: Theory, Methods \& Applications, vol. 75, no. 2, pp. 670-683, 2012.

[15] Y. Gao, Y. Xia, X. Yuan, and P. J. Y. Wong, "Linearization of nonautonomous impulsive system with nonuniform exponential dichotomy," Abstract and Applied Analysis, vol. 2014, Article ID 860378, 7 pages, 2014. 


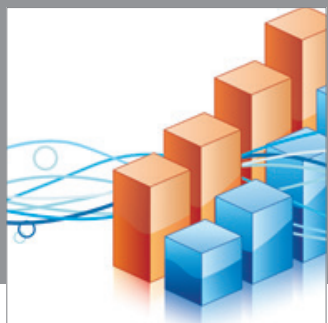

Advances in

Operations Research

mansans

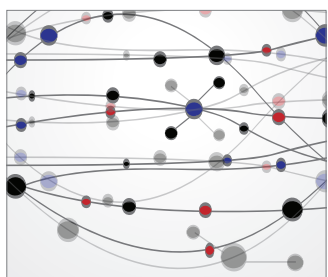

The Scientific World Journal
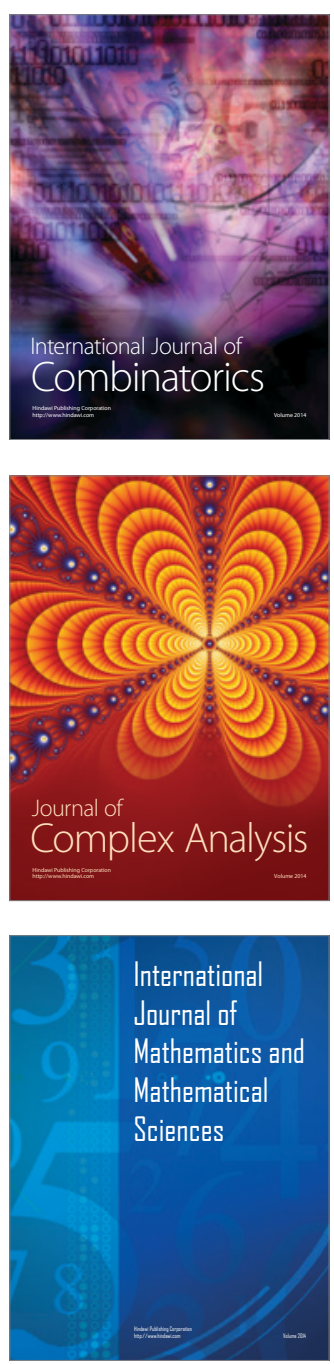
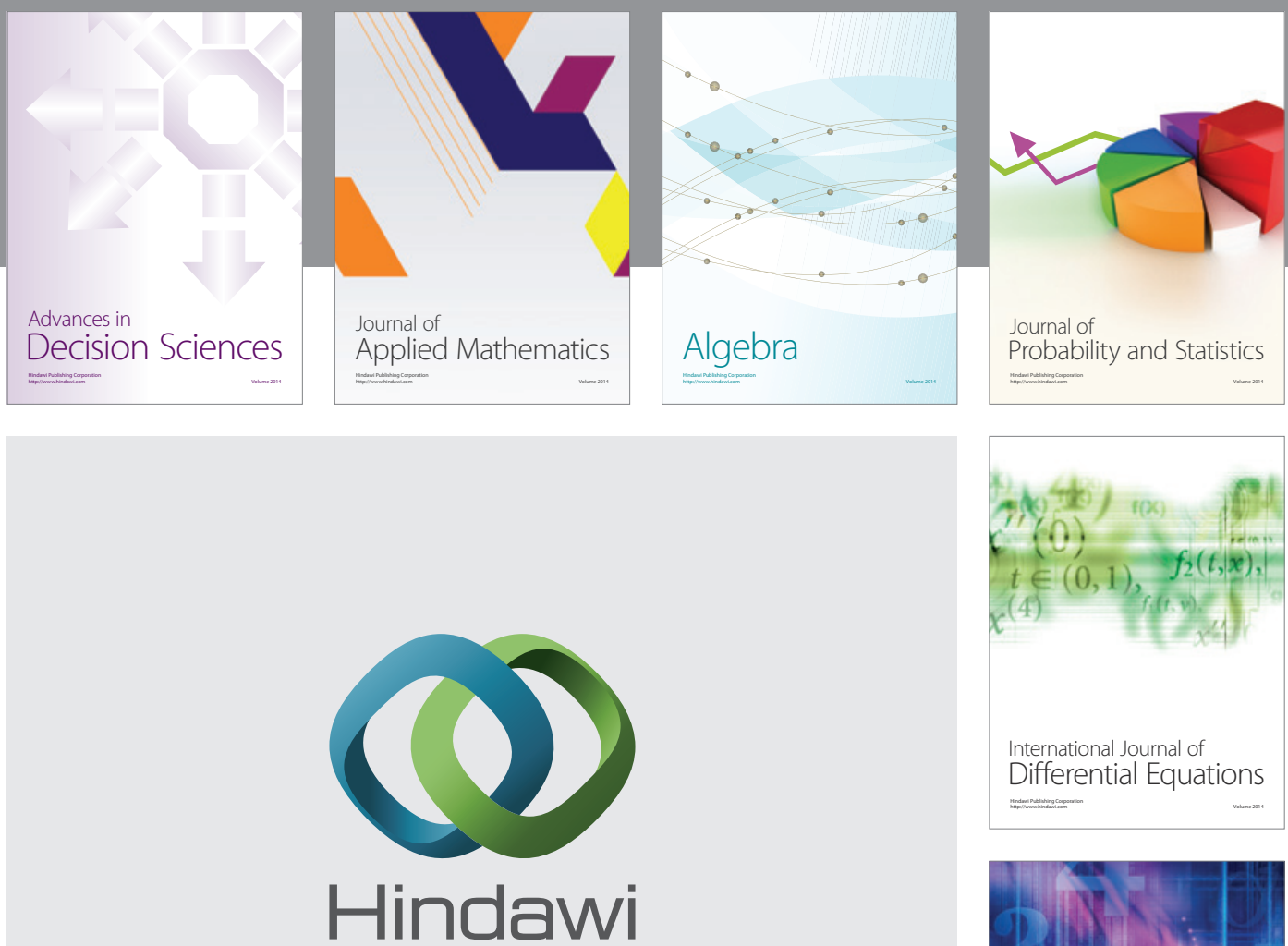

Submit your manuscripts at http://www.hindawi.com
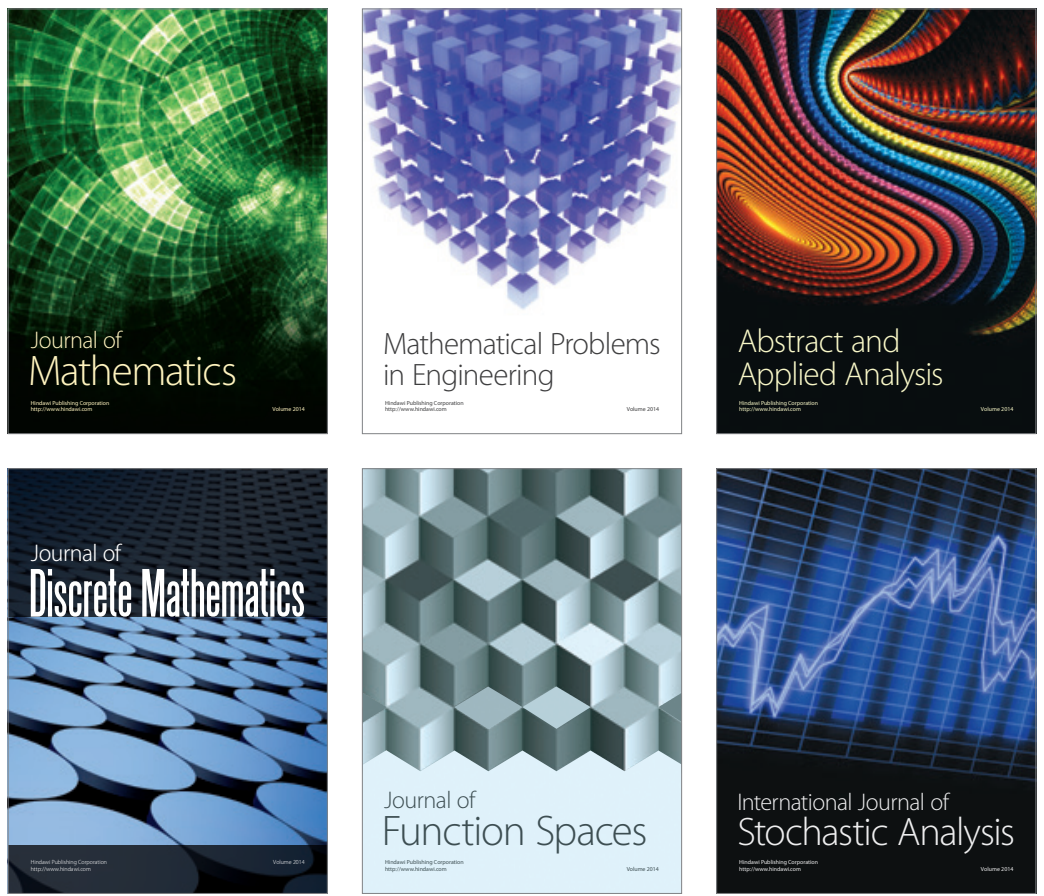

Journal of

Function Spaces

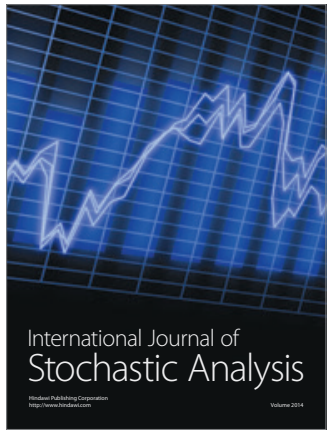

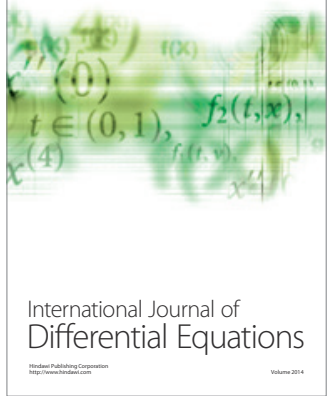
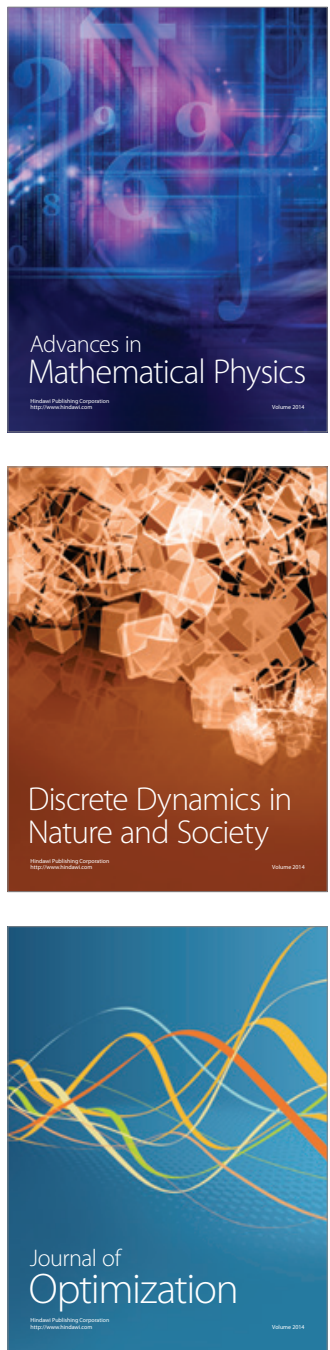